\title{
Biomarkers of Neutrophil-Mediated Glutathione and Protein Oxidation in Tracheal Aspirates From Preterm Infants: Association With Bacterial Infection
}

\author{
D. TIM HARWOOD, BRIAN A. DARLOW, FOOK-CHOE CHEAH, NICOLETTE MCNEILL, PATRICIA GRAHAM, \\ AND CHRISTINE C. WINTERBOURN
}

\begin{abstract}
Departments of Pathology [D.T.H., C.C.W.] and Paediatrics [B.A.D., N.M., P.G.], University of Otago Christchurch, Christchurch 8140, New Zealand; Department of Paediatrics [F.-C.C.], Universiti Kebangsaan Malaysia Medical Centre Jalan Yaacob Latif, Kuala Lumpur 56000, Malaysia
\end{abstract}

\begin{abstract}
Bronchopulmonary dysplasia is associated with neutrophil infiltration into the lungs and oxidative injury. However, the pathological importance of neutrophil oxidants is still not clear. Nosocomial pneumonia is also implicated, but the evidence is limited, in part because of the difficulty of distinguishing genuine infection from bacterial colonization. Good biomarkers of neutrophil oxidant activity and lung infection are needed. We tested whether glutathione sulfonamide, a product of glutathione oxidation by myeloperoxidase-derived hypochlorous acid $(\mathrm{HOCl})$ and a potential new neutrophil oxidant biomarker, is detectable in endotracheal aspirates from ventilated preterm infants. As infectious organisms stimulate neutrophils to generate $\mathrm{HOCl}$, we determined whether levels of $\mathrm{HOCl}$-specific biomarkers were increased in samples that were bacterial culture positive. Glutathione sulfonamide was detected in 66 of 87 endotracheal aspirate samples. Levels correlated with myeloperoxidase activity and another $\mathrm{HOCl}$-specific marker, chlorotyrosine. Median levels of glutathione sulfonamide (4-fold) and other biomarkers (2-fold) were significantly higher in culture positive aspirates. Staphylococcus epidermidis, a frequent colonizer, was associated with glutathione sulfonamide levels no different from those in negative samples. Glutathione sulfonamide showed good sensitivity and specificity for detecting bacterial growth and has promise for detecting lung infection. (Pediatr Res 69: 28-33, 2011)
\end{abstract}

$\mathrm{B}$ ronchopulmonary dysplasia (BPD), otherwise known as neonatal chronic lung disease, remains a major problem in neonatology and child health (1). A large body of evidence implicates both inflammation and oxidative injury in BPD (2-7) with likely sources of oxidative damage including neutrophils and macrophages attracted to the lungs by inflammatory mediators or to combat infection. Neutrophils accumulate early within the lungs of ventilated infants and persistent neutrophil elevation in endotracheal (ET) aspirates is associated with the development of BPD $(8,9)$. When stimulated, they generate reactive oxygen species including hypochlorous acid $(\mathrm{HOCl})$, the only physiological source of which is the neutrophil enzyme, myeloperoxidase (MPO) (10). Active

Received May 19, 2010; accepted August 26, 2010

Correspondence: Christine Winterbourn, Ph.D., Department of Pathology, University of Otago Christchurch, PO Box 4345, Christchurch, New Zealand; e-mail christine.winterbourn@otago.ac.nz

Supported by the Child Health Research Foundation, Lotteries Health Research, the Health Research Council of New Zealand, and the National Research Centre for Growth and Development.
MPO and evidence of $\mathrm{HOCl}$ production have been observed in the lungs of infants who develop BPD $(4,5)$. However, further investigation is required to establish whether neutrophil oxidants have a causative role.

$\mathrm{HOCl}$ is too short lived to measure directly in biological material. Therefore, it is necessary to measure reaction products as biomarkers (10). One established specific marker is 3-chlorotyrosine (Cl-Tyr), formed by its reaction with tyrosine residues in proteins (11). Another promising new candidate biomarker for $\mathrm{HOCl}$ is glutathione sulfonamide (GSA), a stable oxidation product of reduced glutathione (GSH) formed by condensation of the oxidized cysteine with the amine group of the $\gamma$-glutamyl residue $(12,13)$. As GSH is a more favored target than tyrosine for HOCl, GSA may have advantages over $\mathrm{Cl}$-Tyr as a biomarker. Oxidation of intracellular and epithelial lining fluid GSH would be expected if $\mathrm{HOCl}$ is generated in the lungs and GSA should be produced, in addition to glutathione disulfide (GSSG). We have developed a sensitive method for detecting GSA in lung lavage fluid (14), but quantitative measurements in clinical samples have not been reported. Also, it has not been established whether GSA is a useful biomarker of MPO activity and neutrophil activation.

Infection causes the sequestration and activation of neutrophils at inflammatory sites, and several studies have shown that systemic sepsis is a risk factor for BPD (2,15-17). However, diagnosis of lung infection in ventilated infants is difficult, and the relationship between organisms cultured from ET aspirates and BPD is less clear $(18,19)$. Some organisms such as coagulase-negative Staphylococci may merely colonize the ET tube and upper airways and elicit little host response. The role of Ureaplasma spp. in the preterm lung is also controversial $(2,20,21)$. One way of detecting lung infection could be to measure markers of neutrophil activation. Pathogenic but not colonizing bacteria should elicit a neutrophil response that involves generation of reactive oxygen species. As $\mathrm{HOCl}$ is an oxidant largely restricted to

\footnotetext{
Abbreviations: BPD, bronchopulmonary dysplasia; Cl-Tyr, 3-chlorotyrosine; ET, endotracheal; GSA, glutathione sulfonamide; GSH, reduced glutathione; GSSG, glutathione disulfide; MPO, myeloperoxidase; ROC, receiver operating characteristic; VAP, ventilator-associated pneumonia
} 
neutrophils, we hypothesized that neutrophil activity could be assessed by measuring biomarkers of $\mathrm{HOCl}$ production.

We aimed first to establish whether GSA is detectable in ET aspirates from ventilated preterm infants at risk of BPD and whether it reflects neutrophil activity. Second, we explored whether neutrophil-derived oxidative markers, GSA, Cl-Tyr, and MPO, provide an index of infection status by examining their relationship to the growth of bacterial species cultured from the aspirates.

\section{METHODS}

Study group. All ventilated infants $<32$ wk gestation admitted to the regional neonatal intensive care unit at Christchurch Women's Hospital between March 2001 and October 2002 or between July 2004 and May 2006 were eligible for this observational study. The parents of infants who remained intubated for $>48 \mathrm{~h}$ gave written consent to use samples from routine ET aspiration for research. Infants were cared for according to the standard unit protocols. The study was approved by the Upper South Regional Ethics Committee of the New Zealand Ministry of Health. ET suctioning was performed only when clinically required to remove secretions. From a large bank of archival samples collected at various times after birth, we selected those that met the criteria of i) containing sufficient material for analysis and ii) having an aspirate with known culture status collected within $24 \mathrm{~h}$. Samples from the later cohort (87 aspirates from 38 infants) were analyzed for GSA, MPO, and Cl-Tyr. The earlier cohort (76 aspirates from 40 infants) was analyzed for Cl-Tyr only, because the GSA assay was not then available.

Sampling procedure. A dry ET shallow suctioning procedure was used, with instillation of saline if necessary (4). Secretions collected in suction catheters were stored at $4{ }^{\circ} \mathrm{C}$ for up to $12 \mathrm{~h}$ before flushing with PBS. For biomarker analyses, samples from each day were pooled. Supernatants were separated from cellular material and stored at $-80^{\circ} \mathrm{C}$. An aspirate collected from each infant on alternate days was sent for bacterial culture when sufficient material was available. Confirmed culture results were classified as positive, in the presence of at least moderate growth of a single or mixed organisms (more than one quadrant of an agar plate), or negative.

Aspirates collected during the first week of life and confirmed as bacterial culture negative were classified according to whether or not the infant was positive for Ureaplasma urealyticum or other Ureaplasma spp. using the Mycoplasma Duo kit (Bio-Rad, Marnes La Coquette, France). These samples were analyzed for MPO and Cl-Tyr. An average of two samples was analyzed from each infant.

Ventilator-associated pneumonia. We retrospectively reviewed clinical data corresponding with the days ET aspirates were obtained to assess whether the infant was thought to have at least possible ventilator-associated pneumonia (VAP) (22). Criteria used were a) worsening oxygenation as judged by a $10 \%$ increase in the median hourly recorded fraction of inspired oxygen over $24 \mathrm{~h}$ compared with the previous day, b) a radiological report of probable pneumonic consolidation, c) at least three of i) raised C-reactive protein $>10 \mathrm{mg} / \mathrm{L}$; ii) raised immature: total neutrophil ratio $(>0.15)$; iii) temperature instability with no other cause; and iv) a clinical decision to commence antibiotics.

Biochemical analyses. Total protein was measured using the BioRad detergent-compatible assay kit (Hercules, CA), and urea using the QuantiChrom Urea Assay kit (DIUR-500) from BioAssay Systems (Hayward, CA). MPO (total peroxidase) activity was measured using 3,3',5,5'-tetramethybenzidine (4). Aspirate protein was hydrolyzed and analyzed for tyrosine and Cl-Tyr by gas chromatography-mass spectrometry (using a Hewlett Packard 6890 Gas Chromatograph equipped with a 30-m DB17 capillary column and interfaced to a Hewlett Packard 5973 Mass Spectrometer) with isotopically labeled internal standards (5). Results are expressed as Cl-Tyr residues per million Tyr residues $(\mu \mathrm{mol} / \mathrm{mol})$ and have been corrected for artifactual tyrosine chlorination (typically $\sim 10 \mu \mathrm{mol} / \mathrm{mol}$ ).

GSA was analyzed by stable isotope dilution liquid chromatographytandem mass spectrometry assay (LC-MS/MS) using a Thermo Finnigan LCQ Deca XP Plus Ion Trap mass spectrometer (San Jose, CA) coupled to a Thermo Hypercarb column as described (14). Aliquots of standards $(0.2 \mathrm{~mL})$ or ET aspirates (a volume containing $50 \mu \mathrm{g}$ total protein made up to $0.2 \mathrm{~mL}$ ) mixed with isotopically labeled GSA (10 $\mu \mathrm{L}$ of $2 \mu \mathrm{M}$ stock solution) as internal standard. Ice-cold ethanol $(0.8 \mathrm{~mL})$ was added and precipitated protein pelleted. Supernatants were aspirated, dried under vacuum. Residues were redissolved in $200 \mu \mathrm{L}$ of PBS, and $50 \mu \mathrm{L}$ was injected onto the column. GSA was prepared by treating GSH with reagent $\mathrm{HOCl}$ in phosphate buffer
Table 1. Clinical details of study infants

\begin{tabular}{lcc}
\hline & Full cohort & GSA group \\
\hline$N \quad$ & 78 & 38 \\
Sex: M/total (\%) & $45 / 76(59)$ & $22 / 36(61)$ \\
Gestational age (wk), median & $25(22-32)$ & $26(22-32)$ \\
$\quad$ (range) & & \\
Birthweight (g), median & $855(385-1730)$ & $972(385-1780)$ \\
$\quad$ (range) & & \\
Died & 4 & 4 \\
BPD*, $n(\%)$ & $41 / 76(54)$ & $17 / 32(53)$ \\
Total aspirates $\dagger$ & 163 & 87 \\
No. with positive culture & 100 & 63 \\
$\quad$ S. epidermidis & 26 & 15 \\
Other organism $\neq$ & 74 & 48 \\
Sampling d, median & $10(1-67)$ & $13(2-67)$ \\
$\quad$ (range) & & \\
\hline
\end{tabular}

* BPD defined as the requirement for supplementary oxygen at $36 \mathrm{wk}$ postmenstrual age.

$\dagger$ Note that some infants provided more than one aspirate.

$\$$ Positive culture represents at least moderate growth of single or mixed organisms. Organisms cultured include S. aureus, E. coli, Acinetobacter, Enterococcus, Enterobacter, Pseudomonas, Mycoplasma, Serratia, Citrobacter, Moditrobacter, Stenotrophomonas, and Streptococcus spp., S. epidermidis and Candida spp.

Table 2. Summary of biochemical analyses on ET aspirates from very preterm infants

\begin{tabular}{lccc}
\hline & $n$ & Median & IQ range \\
\hline GSA (pmol/mg urea)* & 87 & 60 & $0-300$ \\
GSA (pmol/mg protein) & 87 & 1.8 & $0-5.6$ \\
MPO (nmol/mg urea)*† & 87 & 1.2 & $0.6-2.6$ \\
MPO (pmol/mg protein) & 87 & 31 & $16-62$ \\
3-Cl-Tyr $(\mu \mathrm{mol} / \mathrm{mol}$ Tyr $)$ & 163 & 104 & $53-205$ \\
\hline
\end{tabular}

* Correcting values for variations in circulating urea concentration (23) was not possible as matching blood samples were not available.

$\dagger$ Although total peroxidase activity was measured, the majority of the activity in ET aspirates has been established as due to MPO and not lacto- or eosinophil peroxidase (5).

(100 mM, pH 7.4), with the internal standard generated using [glycine $\left.1,2-{ }^{13} \mathrm{C}_{2},{ }^{15} \mathrm{~N}\right]-\mathrm{GSH}$. Labeled and unlabeled GSA were purified and used to construct a calibration curve based on mass (14). GSA was stable in buffer or aspirates for at least a year at $-20^{\circ} \mathrm{C}$. The detection level in the extracts was $0.4 \mathrm{nM}$.

\section{RESULTS}

Clinical details are given in Table 1. The full cohort of 78 infants who provided ET samples for Cl-Tyr was closely matched for gender, gestational age, birthweight, and outcome to the subgroup of 38 infants whose samples were also analyzed for MPO and GSA.

GSA detection and association with MPO and Cl-Tyr. GSA was detectable in the majority of the 87 ET aspirates analyzed, with levels greater than the detection limit of the assay in 66 (76\%) of the aspirates (Table 2). MPO and Cl-Tyr content of aspirate protein were also measured. Because samples were collected as part of standard treatment, variable dilution was inevitable. Therefore, GSA and MPO levels are expressed relative to the urea concentration in each aspirate. GSA correlated positively with Cl-Tyr $\left(r_{\mathrm{s}}=0.43, p<0.001\right.$ Fig. $1 A)$ and MPO $\left(r_{\mathrm{s}}=0.59, p<0.001\right.$ Fig. $\left.1 B\right)$, and as previously reported (5), there was a significant association 

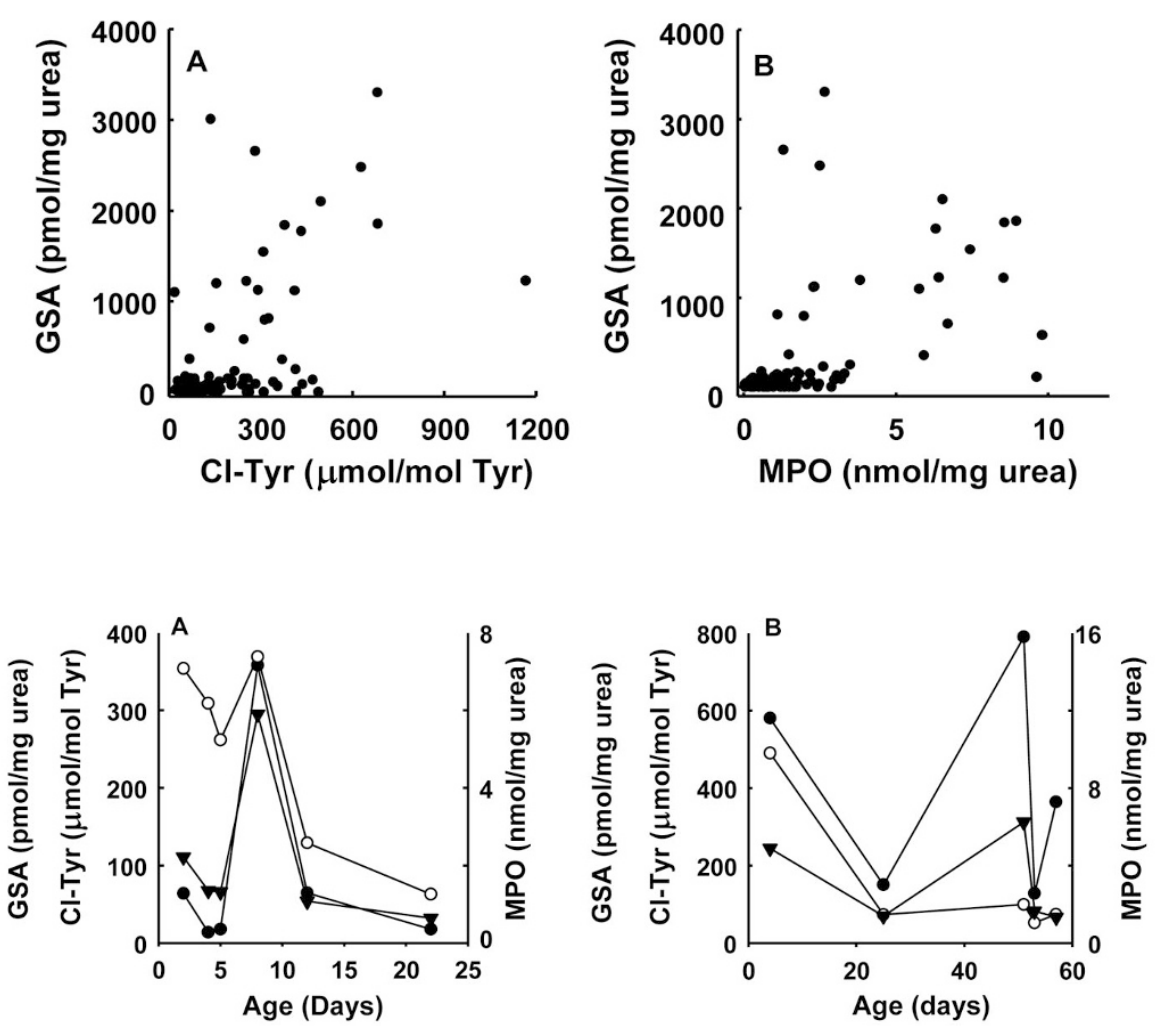

Figure 2. Temporal changes in GSA (-), Cl-Tyr $(\bigcirc)$, and MPO activity $(\mathbf{\nabla})$ in ET aspirates from two infants $(A$ and $B$ ) who provided multiple samples.
Figure 1. Association of GSA levels with $(A)$ Cl-Tyr and $(B)$ MPO activity in ET aspirates. Statistical analysis was by Spearman rank correlation with $p<0.001$ in each case. In support of the results based on urea, similar correlation coefficients and levels of significance were seen when the parameters were related to total protein concentration (data not shown).
Table 3. Biomarker levels in aspirates according to culture status

\begin{tabular}{llll}
\hline \multirow{2}{*}{ Biomarker } & \multicolumn{2}{c}{ Median (IQ range) } & \\
\cline { 2 - 3 } & Culture positive* & Culture negative & \multicolumn{1}{c}{$p$} \\
\hline GSA (pmol/mg urea) & $127(44-774)$ & $28(20-64)$ & $<0.001$ \\
Cl-Tyr $(\mu \mathrm{mol} / \mathrm{mol}$ Tyr) & $134(64-254)$ & $67(39-144)$ & $<0.001$ \\
MPO (nmol/mg urea) & $1.49(0.65-3.10)$ & $0.86(0.36-1.49)$ & 0.009 \\
\hline
\end{tabular}

Results are for 63 positive and 24 negative aspirates (GSA and MPO) and for 100 positive and 63 negative aspirates for Cl-Tyr. Groups were compared using the Mann-Whitney rank sum test. Significant differences between the groups were also apparent when GSA and MPO were expressed relative to protein concentration.

* For any bacterial species (see Table 1).

between Cl-Tyr and MPO $\left(r_{\mathrm{s}}=0.46, p<0.001\right)$. As shown for two individuals who provided multiple aspirates, MPO, GSA, and Cl-Tyr levels fluctuated over time and with few exceptions, followed each other closely (Fig. 2). This is further evidence that the biomarkers are related. It also suggests that they reflect oxidative/inflammatory stresses that vary over time and that they do not accumulate.

Relationship between aspirate culture status and biomarker levels. ET aspirates were classified according to culture status. Those with a positive culture for any organism had significantly elevated levels of GSA, Cl-Tyr, and MPO compared with culture negative samples (Table 3 ). Gestational age and birthweight appeared not to be confounders for these relationships as $t$-test analysis of 22 paired negative and positive aspirates from the same infant also showed higher Cl-Tyr levels in those that were culture positive (median 176 versus $68 \mu \mathrm{mol} / \mathrm{mol} \mathrm{Tyr}, p=0.012$ ). The potential for the biomarkers to distinguish infection from colonization was assessed by further stratification into aspirates that were positive for Staphylococcus epidermidis (S. epidermidis), which is more likely to be a colonizer, and those positive for more pathogenic organisms (as listed in Table 1). Differences between the culture positive and negative groups were greater when aspirates positive for S. epidermidis were excluded (Fig. 3). This was particularly notable for GSA. Levels in the $S$. epidermidis and negative groups were no different, and their median values were 5-fold lower than for the group that cultured positive for other organisms. A similar but less marked trend was observed for MPO, but for Cl-Tyr, the $S$. epidermidis group gave intermediate values that were significantly higher than for negative samples.

The abilities of the three biomarkers to discriminate culture positive aspirates from those that were either culture negative or positive for S. epidermidis was examined by constructing receiver-operating characteristic (ROC) curves. GSA exhibited good discrimination, with an area under curve (AUC) of 0.80 and a cutoff of $120 \mathrm{pmol} / \mathrm{mg}$ urea giving a specificity of $67 \%$ and a false positive rate of $10 \%$ (Fig. 4). MPO (45\% specificity and $15 \%$ false positives above a cutoff of 1 $\mathrm{nmol} / \mathrm{mg}$ urea, AUC 0.71) and Cl-Tyr (36\% specificity and $12 \%$ false positives at a cutoff of $300 \mu \mathrm{mol} / \mathrm{mol} \mathrm{Tyr}$, AUC 0.66 ) were also discriminatory but less so.

There were only five instances where a radiologist independently reported that the chest $\mathrm{x}$-ray at the time of sampling showed "probable pneumonia." Associated ET aspirates were all positive for organisms other than S. epidermidis. Three of these infants had VAP based on the clinical criteria described above (and no other cases met these criteria). For four of the 

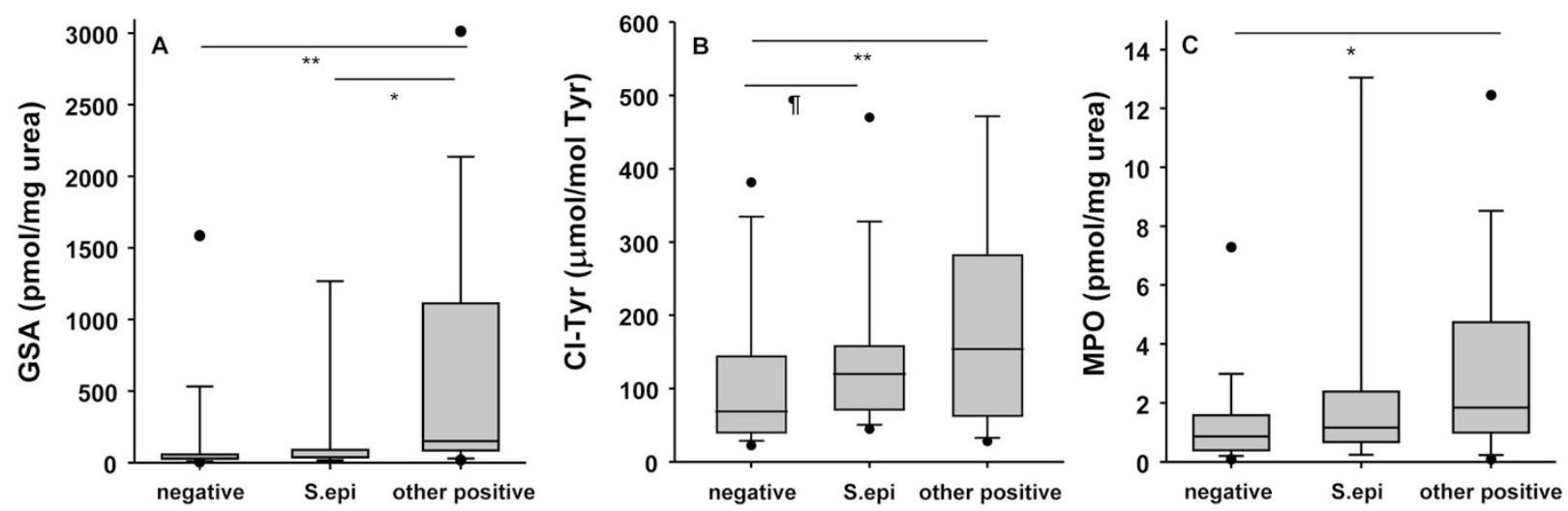

Figure 3. (A) GSA, $(B)$ Cl-Tyr, and $(C)$ MPO levels in culture negative aspirates ( $n=24$ for GSA and MPO, $n=63$ for Cl-Tyr), those positive for $S$. epidermidis ( $n=15$ for GSA and MPO, $n=26$ for Cl-Tyr), and those positive for other organisms ( $n=63$ for GSA and MPO, $n=74$ for Cl-Tyr). Box plots show medians and IQ ranges with bars and symbols (-) representing 90\% and 95\% points, respectively. Medians for the negative group are in Table 3. For groups positive for S. epidermidis and other organisms, respectively, medians (IQ ranges) are: GSA 37 (27-85) and 147 (56-1108) pmol/mg urea; for MPO 0.74 (0.63-1.64) and $1.62(0.93-3.65) \mathrm{nmol} / \mathrm{mg}$ urea. Differences between groups were assessed using the Mann-Whitney rank sum test; $* * p<0.001,{ }^{*} p=0.003, \mathbb{I} p=0.019$.

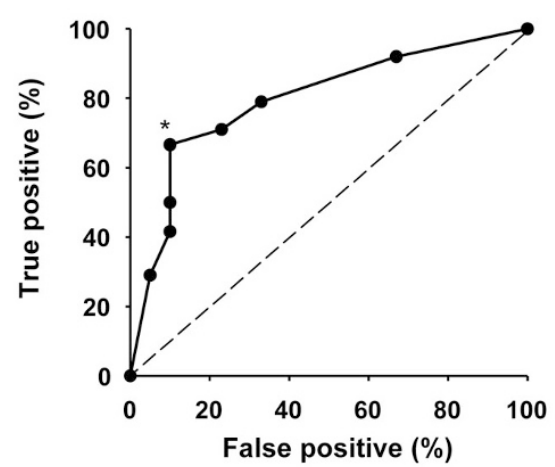

Figure 4. ROC curve for GSA comparing samples that were culture negative or positive for $S$. epidermidis with all other culture positive samples. *120 $\mathrm{pmol} / \mathrm{mg}$ urea cutoff.

five aspirates, all three biomarkers were above the medians for the culture positive group.

Relationship between MPO, Cl-Tyr, and Ureaplasma status. A subgroup of culture negative samples collected in the first week of life were classified into those where the infant cultured positive for Ureaplasma spp. during that time (median gestational age and birthweight $24 \mathrm{wk}$ and $680 \mathrm{~g}$, respectively) and those that were Ureaplasma negative ( $27 \mathrm{wk}, p=$ 0.002 and $867 \mathrm{~g}, p=0.045$ ). For the 109 samples analyzed for MPO, the 27 from Ureaplasma positive infants had median (range) levels of 14.3 (4.4-92) $\mathrm{pmol} / \mathrm{mg}$ protein compared with $14.9(0.6-171) \mathrm{pmol} / \mathrm{mg}$ for the 82 negative infants. Cl-Tyr was analyzed in 42 samples, with median (range) levels of $129(30-208) \mu \mathrm{mol} / \mathrm{mol}$ Tyr for the 11 samples from infants with a positive Ureaplasma response and 147 (41518) $\mu \mathrm{mol} / \mathrm{mol}$ Tyr for the 31 negative samples. There was no difference between the groups for either parameter. The GSA assay was not available when these samples were analyzed.

Culture status and respiratory outcome. We examined whether the infection data related to BPD by classifying the infants as culture positive if they had had at least one culture positive aspirate at any time (48 infants) or culture negative
(21 infants). Four died or moved away before 36 wk postmenstrual age (when BPD status was assessed). Seventy-one percent of the culture positive infants developed BPD compared with $29 \%$ of the culture negative infants $(p=0.002)$. However, the data are confounded because the median day of sampling for culture negative infants was $3 \mathrm{~d}$ compared with $14 \mathrm{~d}$ for those with positive samples. To avoid bias toward sicker babies being intubated longer, the data were reanalyzed for aspirates collected in the first $2 \mathrm{wk}$ of life. All the samples from culture negative infants were collected within that time but the number of culture positive infants dropped to 23 , with median sampling $\mathrm{d} 4$. BPD was still higher in the positive group (57\%), but the difference was no longer significant.

\section{DISCUSSION}

GSA as an oxidative biomarker. To understand the role of reactive oxidants in disease pathology, specific biomarkers are needed to quantify and identify the nature and source of the oxidant. Oxidant production by neutrophils can be identified because these are the only cells that contain MPO and generate $\mathrm{HOCl}$ (with a minor contribution from monocytes/ macrophages). The antioxidant GSH is present at high concentrations within cells and in epithelial lining fluid of the lung (23) and is among the most reactive biomolecules with $\mathrm{HOCl}$. As GSA is produced in significant amounts in this reaction but scarcely at all with other oxidants (12), it is an attractive biomarker candidate. Here, we demonstrate that GSA can be measured quantitatively in clinical samples and show that it is detectable in a majority of ET aspirates from ventilated preterm infants. Positive correlations with MPO and Cl-Tyr support the premise that GSA is derived from MPO activity, and our data demonstrate its potential as a biomarker for neutrophil/MPO activity.

Until now, Cl-Tyr has been the preferred biomarker of $\mathrm{HOCl}$ and MPO activity (10). It has been detected in a number of inflammatory situations, including the lungs of premature infants and children $(5,24,25)$. However, $\mathrm{HOCl}$ reacts slowly with tyrosine and $\mathrm{Cl}-\mathrm{Tyr}$ accounts for only a small fraction of 
biologically generated $\mathrm{HOCl}$. GSA has the advantage of being derived from a more favored target. Many factors affect biomarker levels, including the availability of the target compound and oxidant, the rate of reaction, physiological stability, and clearance rates. Therefore, measurements of multiple biomarkers may provide useful complementary information.

GSSG formation is commonly used as an indicator of oxidative stress. However, a range of oxidants including $\mathrm{HOCl}$ can convert GSH to GSSG. The advantage of GSA is that it identifies $\mathrm{HOCl}$ as the oxidant and neutrophils as its most likely source. We did not measure GSH or GSSG as this requires immediate treatment of the aspirates to prevent $e x$ vivo oxidation. However, with facile adaptation the method used here could be used to measure all three species (14).

Oxidative injury and BPD. Oxidative damage is widely implicated in the pathological changes leading to BPD (7), with poor respiratory outcome associated with elevated levels of oxidative markers including Cl-Tyr in ET aspirate protein $(4-6,26)$. Although the focus of this study was not on outcome, our GSA data extend previous findings that neutrophils produce $\mathrm{HOCl}$ in the lungs of these infants. $\mathrm{HOCl}$ is a highly reactive oxidant that damages cells and inactivates proteins such as $\alpha_{1}$ antitrypsin and surfactant proteins $(10,27,28)$. GSH provides important antioxidant defense, especially in infants with respiratory distress (26). Our finding that lung GSH reacts with $\mathrm{HOCl}$ may indicate that it may not only play a protective role but also imply a degree of antioxidant depletion.

Neutrophil oxidant biomarkers and infection. Inflammation is a well-established contributor to BPD, and there is strong evidence that systemic sepsis $(2,15-17)$ and nosocomial airway infection $(19,29,30)$ are linked to lung injury. Our findings also suggest an association between positive culture and a higher incidence of BPD. However, the relationship with infection is not clearcut (18), and improved methods for assessing infection are desirable. On the basis that neutrophils should respond to an infection by generating microbicidal oxidants, we reasoned that biomarkers of these oxidants would provide an index of infection status. Although we do not have ex vivo data to confirm that the neutrophils from the infant lungs had responded to bacterial exposure, our findings that levels of MPO, Cl-Tyr, and GSA were substantially higher in aspirates with confirmed growth than in those that were culture negative support the contention that they were activated. Measurements of Cl-Tyr and MPO extend our previous study (5). The current study shows that GSA may be an even better infection marker and highlights potential clinical applications.

However, positive culture and infection are not synonymous, and a mechanism for distinguishing ET colonization from lung infection is needed. This is often difficult given the chronic changes in the lungs. As S. epidermidis and other commensals are frequently present as colonizers of the ET tube (31), we tested whether our biomarkers could distinguish the presence of $S$. epidermidis from other organisms. When $S$. epidermidis samples were excluded, the difference between culture negative and positive aspirates became more striking, especially for GSA where the median was 5-fold higher for the positive samples. Furthermore, GSA levels in aspirates that cultured positive for S. epidermidis were no different from those in the negative aspirates. MPO activity showed a similar but less striking trend, whereas Cl-Tyr levels were slightly but significantly higher when $S$. epidermidis was present than in negative samples. Therefore, our results are consistent with $S$. epidermidis not usually eliciting a neutrophil response to infection. Studies with isolated cells have shown that suspensions of S. epidermidis can stimulate neutrophils, albeit with a lower response than for Staphylococcus aureus (32). However, when they are adherent and form biofilms, the response is much less. Low biomarker levels could reflect little interaction between neutrophils and adherent S. epidermidis colonizing the tube. However, commensals are not necessarily innocent bystanders and can cause neonatal sepsis $(17,33)$. The possibility that GSA measurements could detect these cases warrants investigation.

VAP is difficult to diagnose in preterm infants with evolving radiological changes of chronic lung disease $(29,34,35)$. The x-rays are often inconclusive, and markers such as C-reactive protein often fluctuate for other reasons and may not respond rapidly to localized infection. Our study did not examine VAP systematically, and we had few infants where sufficient clinical data were collected to assign to this category. Hence, although they generally had oxidative biomarkers levels in the upper range for culture positive samples, a more focused follow-up is needed to assess whether they are diagnostically informative.

The role of Ureaplasma spp. in BPD is much debated $(20,21)$. In baboons (36) and premature infants (37), only a subset of individuals with Ureaplasma colonization have persistent low grade infection and an increased risk of BPD. Our observation that neither MPO nor Cl-Tyr was elevated in ET aspirates that cultured positive for Ureaplasma implies that Ureaplasma stimulates limited neutrophil oxidant production or oxidative injury.

We have shown that three markers of neutrophil oxidant activity are elevated in culture positive ET aspirates. To be of practical use as indices of infection or inflammation, they need to have advantages over other markers. Inflammatory mediators can attract neutrophils without necessarily stimulating them to release oxidants and granule constituents. Therefore, specific oxidation products should be a better index of neutrophil activation in response to an infection than cell counts. Of the biomarkers measured, GSA was the most discriminatory for aspirates that cultured the more pathogenic organisms, with a cutoff of 120 pmol GSA per mg urea identifying about two thirds of culture positive patients with only $10 \%$ false positives. If further investigation shows that GSA analysis can distinguish colonizing organisms or those that give rise to VAP, it would be a valuable addition for assessing lung infection in infants with respiratory distress.

GSA is straightforward to measure in ET aspirates. One limitation is that such samples are not always available from ventilated infants. The assay is also not applicable for infants treated with nasal continuous positive airways pressure. Although this procedure does seem to decrease the incidence and severity of BPD (38), some preterm infants who had no or 
only brief intubation do develop BPD, and there is still need for monitoring infection. The possibility of using urinary GSA or Cl-Tyr to monitor oxidative stress and infection status in these patients needs to be explored.

Acknowledgments. We thank Jennifer Gardener for assisting with sample collection, Rufus Turner for performing some analyses, and Tony Kettle for helpful discussion.

\section{REFERENCES}

1. Kinsella JP, Greenough A, Abman SH 2006 Bronchopulmonary dysplasia. Lancet 367:1421-1431

2. Speer CP 2006 Inflammation and bronchopulmonary dysplasia: a continuing story. Semin Fetal Neonatal Med 11:354-362

3. Jobe AH, Bancalari E 2001 Bronchopulmonary dysplasia: NICHD/NHLBI/ORD workshop summary. Am J Respir Crit Care Med 163:1723-1729

4. Buss IH, Darlow BA, Winterbourn CC 2000 Elevated protein carbonyls, lipid peroxidation products and myeloperoxidase in tracheal aspirates from premature infants. Pediatr Res 47:640-645

5. Buss IH, Senthilmohan R, Darlow BA, Mogridge N, Kettle AJ, Winterbourn CC 2003 3-Chlorotyrosine as a marker of protein damage by myeloperoxidase in tracheal aspirates from preterm infants: association with adverse respiratory outcome. Pediatr Res 53:455-462

6. Varsila E, Pesonen E, Andersson A 1995 Early protein oxidation in the neonatal lung is related to development of chronic lung disease. Acta Paediatr 84:1296-1299

7. Saugstad OD 2003 Bronchopulmonary dysplasia-oxidative stress and antioxidants. Semin Neonatol 8:39-49

8. Ogden BE, Murphy S, Saunders GC, Johnson JD 1983 Lung lavage of newborns with respiratory distress syndrome. Prolonged neutrophil influx is associated with bronchopulmonary dysplasia. Chest $83: 31 \mathrm{~S}-33 \mathrm{~S}$

9. Merritt TA, Stuard ID, Puccia J, Wood B, Edwards DK, Finkelstein J, Shirpiro DL 1981 Newborn tracheal aspirate cytology: Classification during respiratory distress syndrome and bronchopulmonary dysplasia. J Pediatr 98:949-956

10. Winterbourn CC, Kettle AJ 2000 Biomarkers of myeloperoxidase-derived hypochlorous acid. Free Radic Biol Med 29:403-409

11. Domigan NM, Charlton TS, Duncan MW, Winterbourn CC, Kettle AJ 1995 Chlorination of tyrosyl residues in peptides by myeloperoxidase and human neutrophils. J Biol Chem 270:16542-16548

12. Harwood DT, Kettle AJ, Winterbourn CC 2006 Production of glutathione sulfonamide and dehydroglutathione from GSH by myeloperoxidase-derived oxidants and detection using a novel LC-MS/MS method. Biochem J 399:161-168

13. Harwood DT, Nimmo SL, Kettle AJ, Winterbourn CC, Ashby MT 2008 Molecular structure and dynamic properties of a sulfonamide derivative of glutathione that is produced under conditions of oxidative stress by hypochlorous acid. Chem Res Toxicol 21:1011-1016

14. Harwood DT, Kettle AJ, Brennan S, Winterbourn CC 2009 Simultaneous determination of reduced glutathione, glutathione disulphide and glutathione sulphonamide in cells and physiological fluids by isotope dilution liquid chromatography-tandem mass spectrometry. J Chromatogr B Analyt Technol Biomed Life Sci 877:33933399

15. Van Marter LJ, Dammann O, Allred EN, Leviton A, Pagano M, Moore M, Martin C 2002 Chorioamnionitis, mechanical ventilation, and postnatal sepsis as modulators of chronic lung disease in preterm infants. J Pediatr 140:171-176

16. Gonzalez A, Sosenko IR, Chandar J, Hummler H, Claure N, Bancalari E 1996 Influence of infection on patent ductus arteriosus and chronic lung disease in premature infants weighing 1000 grams or less. J Pediatr 128:470-478
17. Liljedahl M, Bodin L, Schollin J 2004 Coagulase-negative staphylococcal sepsis as a predictor of bronchopulmonary dysplasia. Acta Paediatr 93:211-215

18. Groneck P, Schmale J, Soditt V, Stutzer H, Gotze-Speer B, Speer CP 2001 Bronchoalveolar inflammation following airway infection in preterm infants with chronic lung disease. Pediatr Pulmonol 31:331-338

19. Cordero L, Ayers LW, Miller RR, Seguin JH, Coley BD 2002 Surveillance of ventilator-associated pneumonia in very-low-birth-weight infants. Am J Infect Control 30:32-39

20. Viscardi RM, Hasday JD 2009 Role of Ureaplasma species in neonatal chronic lung disease: epidemiologic and experimental evidence. Pediatr Res 65:84R-90R

21. Maxwell NC, Nuttall D, Kotecha S 2009 Does Ureaplasma spp. cause chronic lung disease of prematurity: ask the audience? Early Hum Dev 85:291-296

22. Horan TC, Andrus M, Dudeck MA 2008 CDC/NHSN surveillance definition of health care-associated infection and criteria for specific types of infections in the acute care setting. Am J Infect Control 36:309-332

23. Cantin AM, North SL, Hubbard RC, Crystal RG 1987 Normal alveolar epithelial lining fluid contains high levels of glutathione. J Appl Physiol 63:152-157

24. Kettle AJ, Chan T, Osberg I, Senthilmohan R, Chapman AL, Mocatta TJ, Wagener JS 2004 Myeloperoxidase and protein oxidation in the airways of young children with cystic fibrosis. Am J Respir Crit Care Med 170:1317-1323

25. Van der Vliet A, Nguyen MN, Shigenaga MK, Eiserich JP, Marelich GP, Cross CE 2000 Myeloperoxidase and protein oxidation in cystic fibrosis. Am J Physiol Lung Cell Mol Physiol 279:L537-L546

26. Cooke RW, Drury JA 2005 Reduction of oxidative stress marker in lung fluid of preterm infants after administration of intra-tracheal liposomal glutathione. Biol Neonate 87:178-180

27. Matheson NR, Travis J 1985 Differential effects of oxidizing agents on human plasma alpha 1-proteinase inhibitor and human neutrophil myeloperoxidase. Biochemistry 24:1941-1945

28. Manzanares D, Rodriguez-Capote K, Liu S, Haines T, Ramos Y, Zhao L, DohertyKirby A, Lajoie G, Possmayer F 2007 Modification of tryptophan and methionine residues is implicated in the oxidative inactivation of surfactant protein B. Biochemistry 46:5604-5615

29. Cordero L, Ayers LW, Davis K 1997 Neonatal airway colonization with gramnegative bacilli: association with severity of bronchopulmonary dysplasia. Pediatr Infect Dis J 16:18-23

30. Coalson JJ, Gerstmann DR, Winter VT, deLemos RA 1991 Bacterial colonization and infection studies in the premature baboon with bronchopulmonary dysplasia. Am Rev Respir Dis 144:1140-1146

31. von Eiff C, Peters G, Heilmann C 2002 Pathogenesis of infections due to coagulasenegative staphylococci. Lancet Infect Dis 2:677-685

32. Nilsdotter-Augustinsson A, Wilsson A, Larsson J, Stendahl O, Ohman L, LundqvistGustafsson H 2004 Staphylococcus aureus, but not Staphylococcus epidermidis, modulates the oxidative response and induces apoptosis in human neutrophils. APMIS 112:109-118

33. Sohn AH, Garrett DO, Sinkowitz-Cochran RL, Grohskopf LA, Levine GL, Stover BH, Siegel JD, Jarvis WR 2001 Prevalence of nosocomial infections in neonatal intensive care unit patients: results from the first national point-prevalence survey. J Pediatr 139:821-827

34. Torres A, Ewig S 2004 Diagnosing ventilator-associated pneumonia. N Engl J Med 350:433-435

35. Garland JS, Uhing MR 2009 Strategies to prevent bacterial and fungal infection in the neonatal intensive care unit. Clin Perinatol 36:1-13

36. Yoder BA, Coalson JJ, Winter VT, Siler-Khodr T, Duffy LB, Cassell GH 2003 Effects of antenatal colonization with Ureaplasma urealyticum on pulmonary disease in the immature baboon. Pediatr Res 54:797-807

37. Castro-Alcaraz S, Greenberg EM, Bateman DA, Regan JA 2002 Patterns of colonization with Ureaplasma urealyticum during neonatal intensive care unit hospitalizations of very low birth weight infants and the development of chronic lung disease. Pediatrics 110:e45

38. Verder H, Bohlin K, Kamper J, Lindwall R, Jonsson B 2009 Nasal CPAP and surfactant for treatment of respiratory distress syndrome and prevention of bronchopulmonary dysplasia. Acta Paediatr 98:1400-1408 